\title{
Calculation of Bulk Acoustic Wave Propagation Velocities in Trigonal Piezoelectric Smart Materials
}

\author{
Abo-el-nour N. Abd-alla ${ }^{1,2, *}$ and Nadia A. Askar ${ }^{1}$ \\ ${ }^{1}$ Department of Mathematics, Faculty of Science, Jazan University, Jazan, Saudi Arabia \\ ${ }^{2}$ Department of Mathematics, Faculty of Science, Sohag University, Sohag 82524, Egypt
}

Received: 18 Jul. 2013, Revised: 20 Oct. 2013, Accepted: 21 Oct. 2013

Published online: 1 Jul. 2014

\begin{abstract}
The goal of this paper is to determine the bulk acoustic wave (BAW) propagation velocities (quasi-longitudinal, quasi-shear vertical and quasi-shear horizontal) in two important piezoelectric smart materials, Lithium Niobate $\left(\mathrm{LiNbO}_{3}\right)$ and Lithium Tantalate $\left(\mathrm{LiTaO}_{3}\right)$. To determine the BAW propagation velocities, the BAW elemental equations are deduced. The BAW velocities are calculated for each direction by solving the Christoffel's equation systematically based on the theory of acoustic waves in anisotropic solids exhibiting piezoelectricity. The modification of the BAW velocities by the piezoelectric effect are calculated and graphically compared with the velocities in the corresponding non-piezoelectric materials. Furthermore, the electromechanical coupling factors are defined and investigated. The results obtained in this study can be applied to signal processing, sound systems and wireless communication in addition to the improvement of surface acoustic wave (SAW) devices and military defense equipment.
\end{abstract}

Keywords: Bulk acoustic wave; Lithium Niobate; Lithium Tantalate; Piezoelectric crystal; Christoffel's equation

\section{Introduction}

The class of engineering materials known as "smart materials" or "intelligent materials" has become a major focus of attention. In particular, piezoelastic (piezoelectric) materials have great promise for use in smart structural systems. When an external force acts on a piezoelastic material, the mechanical stresses produce an electric potential within the material. Conversely, when an electric field is applied to a piezoelastic material, stresses are induced. The possibility exists to not only determine the stresses in a piezoelastic material by measuring the electric potential but also control the stresses by the action of an appropriate applied electric field [1].

Waves propagating in an unbounded, homogeneous medium are called bulk acoustic waves (BAW). For higher symmetry crystals, if the propagation direction coincides with the principal axis direction, then the phase velocities can be easily calculated. Nevertheless, for an arbitrary propagation direction, which requires solving the characteristic equation to find the phase velocities (i.e., the corresponding eigenvalues of Christoffel's equation), the computations become complex and cumbersome. So, the theoretical description of bulk elastic waves in anisotropic bodies involves solving algebraic equations of degree three or higher, and obtaining explicit results is only possible for simplified situations due to symmetry or special relations between the material parameters in the model medium. Nevertheless many fundamental properties of bulk waves in anisotropic media became understood through the explicit analysis of various particular illuminating situations that are described by direct calculations (see, e.g. $[2,3,4]$ and $[5])$.

There is increasing interest in the theory of electroacoustic waves in piezoelectric materials poses numerous challenging problems [2]. Much of the interest in the subject is directed towards applications in the areas of signal processing, transducers, radio-frequency resonators, band-pass filters and frequency control $[6,7,8$, $9,10]$ and [11]. From the viewpoint of wave propagation, the piezoelectric problem is closely related to the purely acoustic problem. In fact, it can be shown that, in an infinite homogenous medium, piezoelectric waves propagate in the form of plane waves. Because the velocity of acoustic waves is much smaller than that of electromagnetic waves, in a ratio of $10^{5}$, the electromagnetic field is assumed to propagate

\footnotetext{
*Corresponding author e-mail: Aboelnourabdalla@yahoo.com
} 
instantaneously and the electric field can be derived from a potential, which is called the quasi-static approximation [8].

The theory of acoustic axes in anisotropic materials (crystals) has many different aspects. The main activity in this field was initiated by Khatkevich [12], who derived the general equations determining the directions of degeneracy for crystals of unrestricted anisotropy. The theory developed by Khatkevich was not invariant and the form of its basic equations depended on the choice of coordinate system. So, different versions of the invariant criteria for degeneracy of the phase speeds of bulk waves in crystals were later introduced in [13,14], and [15].

Zhou et al. [16] discussed the acoustic anisotropy of the crystals, and the slowness curves of three bulk waves and surface waves for different planes considering piezoelectric effects were calculated. Alshits et al. [17] presented a short survey of some basic results in the theory of bulk elastic waves in anisotropic media and a series of general properties of phase speed branches was deduced. Ting [18] described some of the properties of anisotropic elastic materials such as orthotropic and hexagonal materials. Additionally, Ting [19] proved that a necessary and sufficient condition exists for the presence of longitudinal and transverse waves in anisotropic elastic materials. Sharma [20,21] studied the propagation of plane harmonic waves in anisotropic elastic and piezo-poroelastic media and presented a new procedure to study the reflection in anisotropic media. Langer and Selberherr [22] developed an analysis and computer program for acoustic wave generation in piezoelectric materials, which takes into account the second-order effects of bulk wave anisotropic materials. Mah and Schmitt [23] demonstrated that many rocks may be considered to exhibit orthorhombic symmetry; they experimentally determined the nine independent elastic coefficients required for their case of study. Recently, Cristini et al. [24] presented a review of wave propagation at the surface of anisotropic media (crystal symmetries). The physics for media of cubic and hexagonal symmetries has been extensively studied based on analytical and semi-analytical methods. Mauritsson et al. [25] derived a hierarchy of dynamic plate equations for a fully anisotropic elastic plate, and the explicit plate equations were presented and compared analytically and numerically to other approximate theories given in the literature. Ostrosablin [26] obtained the general form of the displacement vectors of plane transverse waves in elastic isotropic and anisotropic media, and he determined the eigenmoduli, eigenstates, and engineering constants: bulk moduli, Youngs moduli, Poisson coefficients, shear moduli, and Lame's constants of the closest isotropic materials. The calculations of quasi-longitudinal, quasi-shear vertical and quasi-shear horizontal waves in anisotropic piezoelectric smart materials have been used in studying the phenomena of reflection and refraction wave propagation in many papers, such as: $[27,28,29,30]$ and [31].
In this paper, we attempt to build a mathematical model based on the partial differential equation system in three spatial dimensions consisting of the equations of motion and Poisson's equation. The derivation of this system from the fundamental equations describing acoustic wave propagation in arbitrary piezoelectric materials is described in [8]. By solving the system of equations, we obtained the bulk acoustic wave (BAW) propagation velocities (quasi-longitudinal, quasi-shear vertical and quasi-shear horizontal) for Lithium Niobate $\left(\mathrm{LiNbO}_{3}\right)$ and Lithium Tantalate $\left(\mathrm{LiTaO}_{3}\right)$. The modification of the velocities of wave propagation caused by the piezoelectric effect in those materials is calculated and compared with the corresponding non-piezoelectric materials. These results are presented graphically for comparison. Furthermore, the electromechanical coupling factors are defined and investigated.

\section{The basic equations for the wave motion in piezoelectric media}

The basic wave equation for displacements in an elastic, homogeneous, anisotropic medium is expressed as

$\sigma_{i j, j}=\rho \ddot{u}_{i}$,

where $\rho$ is the mass density of the elastic medium, $\sigma_{i j}$ is the stress tensor and $u_{i}$ is the displacement tensor.

In a piezoelectric material, the mechanical equations of motion and Maxwells equations for the electrical behavior are coupled through two constitutive equations given by:

$\sigma_{i j}=c_{i j k l} S_{k l}-e_{k i j} E_{k}$,

$D_{i}=\varepsilon_{i j} E_{k}+e_{i k l} S_{k l}$,

where $c_{i j k l}(i, j, k, l=1,2,3)$ is the stiffness tensor, $S_{k l}$ is the strain tensor (second rank), $e_{i k l}$ is the third rank piezoelectric tensor, $E_{i}$ is the electric field, $D_{i}$ is the component of electric displacement and $\varepsilon_{i k}$ is the symmetric permittivity matrix.

The components of strain displacement are given by:

$S_{i j}=\frac{1}{2}\left(u_{i, j}+u_{j, i}\right)$.

Because the velocity of acoustic waves are five times smaller in magnitude compared to the velocity of electromagnetic waves, the frequency of the electric field waves can be considered to be small enough to categorize them as quasi-static. In such a case, the curl of the electric field can be assumed to vanish, thereby reducing one of Maxwell's equations to

$$
\vec{\nabla} \wedge \vec{E}=\overrightarrow{0}
$$


thus enabling us to describe the electric field as the negative gradient of the scalar potential given by the following equation:

$E_{i}=-\varphi_{, i}$.

Magnetic fields are rarely of interest in bulk or surface acoustic wave problems and hence the Maxwells equations concerning them are disregarded. The piezoelectric materials are almost perfect insulators, thereby reducing the remaining Maxwell's equation to:

$D_{i, i}=0$.

Substituting the relations for and given by equations (4) and (6), respectively, into the piezoelectric constitutive equations (2) and (3) and henceforth into the wave equation and Maxwell's equation provides a system of coupled wave equations given by:

$$
\begin{aligned}
& c_{i j k l} u_{l, j k}+e_{k j i} \varphi_{j k}=\rho \ddot{u}_{i}, \\
& e_{j k l} u_{l, j k}-\varepsilon_{j k} \varphi_{j k}=0 .
\end{aligned}
$$

\section{Propagation of bulk waves in anisotropic piezoelectric elastic media}

The general solution of the coupled equations of piezoelectricity (8) and (9) for an arbitrary piezoelectric medium may be expressed for the potential $\varphi\left(x_{j}, t\right)$ and the displacement vector $u_{k}\left(x_{j}, t\right)$ in the form:

$u_{k}\left(x_{j}, t\right)=U_{k} \exp \left[i \frac{n_{j} x_{j}}{v}-\omega t\right]$

$\varphi\left(x_{j}, t\right)=\Phi \exp \left[i \frac{n_{j} x_{j}}{v}-\omega t\right]$.

In (10) and (11), plane monochromatic waves are propagated in the direction of vector $n_{j}$, which is perpendicular to the wave front, $v$ is the phase velocity of the wave and $k, j=1,2,3$.

Substituting (10) and (11) into (8) and (9), we obtain a system of linear homogeneous equations with respect to the peak values of $\Phi$ and $U_{k}$ :

$\left(c_{k l p s} n_{l} n_{p}-\rho v^{2} \delta_{k s}\right) U_{s}+e_{s k j} n_{j} n_{s} \Phi=0$,

$e_{s k j} n_{j} n_{s} U_{k}-\varepsilon_{j k} n_{j} n_{k} \Phi=0$.

The system (12) and (13) may be written as follows:

$\left(\Gamma_{k s}-\rho v^{2} \delta_{k s}\right) U_{s}+\gamma_{k} \Phi=0$

$\gamma_{k} U_{k}-\varepsilon^{s} \Phi=0$

where

$$
\begin{aligned}
& \Gamma_{k s}=c_{k l p s} n_{l} n_{p}, \\
& \gamma_{k}=e_{s k j} n_{j} n_{s}, \\
& \varepsilon^{s}=\varepsilon_{j k} n_{j} n_{k} .
\end{aligned}
$$

Eliminating the peak value of the potential $\Phi$ from the system (14) and (15), we have

$\left.\left[\Gamma_{k s}+\frac{\gamma_{k} \gamma_{s}}{\varepsilon^{s}}\right)-\rho v^{2} \delta_{k s}\right] U_{s}=0, \quad k=1,2,3$.

A non-trivial solution of system (17) exists if its determinant (which is so-called Christoffel's equation) is equal to zero. Taking into account tensor $\Gamma_{k s}$, this condition may be written in the form

$\Delta=\left|\begin{array}{ccc}\widetilde{\Gamma}_{11}-\rho v^{2} & \widetilde{\Gamma}_{12} & \widetilde{\Gamma}_{13} \\ \widetilde{\Gamma}_{21} & \widetilde{\Gamma}_{22}-\rho v^{2} & \widetilde{\Gamma}_{23} \\ \widetilde{\Gamma}_{31} & \widetilde{\Gamma}_{32} & \widetilde{\Gamma}_{33}-\rho v^{2}\end{array}\right|=0$,

where

$\widetilde{\Gamma}_{k s}=\Gamma_{k s}+\frac{\gamma_{k} \gamma_{s}}{\varepsilon^{s}}$

Equation (18) allows to determine the velocity of the plane waves propagating along the direction $n_{j}$. As equation (18) is an equation of the third power with respect to $\left(\rho v^{2}\right)$, in the general case for the given direction in a piezoelectric crystal, we have three velocities $\rho v_{j}^{2}$ where $(j=1,2,3)$, which are determined by the positive roots of equation (18). Substituting each of the values $v_{j}$ into system (17), we obtain $U_{s}^{(j)}, \Phi^{(j)}=\frac{\gamma_{k} U_{s}^{(k)}}{\varepsilon^{s}}$. Due to the homogeneity of equation (17), the quantities $U_{s}^{(j)}, \Phi^{(j)}$ are determined up to the multiplication by an arbitrary constant. Thus, the problem of determining the velocity of plane waves propagating along an arbitrary direction $n_{j}$ in a piezoelectric medium is reduced to finding the Eigenvalues $\rho v_{j}^{2}$ and eigenvectors $U_{s}^{(j)}$ of tensor $\widetilde{\Gamma}_{k s}$. Note that due to the symmetry of tensor $\widetilde{\Gamma}_{k s}$ its Eigenvalues will be real quantities, and because the quadratic form of $\Gamma_{k s} U_{k} U_{s}$ is positive, the Eigenvalues $\widetilde{\Gamma}_{k s}$ are also positive. For any of the three plane waves propagating in direction $n_{j}$, the vector of displacement $U^{(j)}$ in the general case will not be parallel or perpendicular to the direction of propagation. However, the wave with vector $U^{(1)}$ forming the smallest angle with the direction $n_{j}$ is usually called quasi-longitudinal, and the two elastic waves with vectors $U^{(2)}, U^{(3)}$ are called quasi-transverse. In addition, the vectors $U^{(1)}, U^{(2)}, U^{(3)}$ are perpendicular to each other. Before we address propagation along the direction linked to the symmetry elements, we first establish the expressions for the $\Gamma_{k s}$ tensor components. Expanding the sum over $k$ and $s$ in the system (16), one can find:

$$
\begin{aligned}
\Gamma_{i l}= & c_{i 11 l} n_{1}^{2}+c_{i 22 l} n_{2}^{2}+c_{i 33 l} n_{3}^{2}+\left(c_{i 12 l}+c_{i 21 l}\right) n_{1} n_{2} \\
& +\left(c_{i 13 l}+c_{i 31 l}\right) n_{1} n_{3}+\left(c_{i 23 l}+c_{i 32 l}\right) n_{2} n_{3}
\end{aligned}
$$


In detail, the six components of $\Gamma_{i l}$ are:

$$
\begin{aligned}
\Gamma_{11}= & c_{11} n_{1}^{2}+c_{66} n_{2}^{2}+c_{55} n_{3}^{2}+2 c_{16} n_{1} n_{2} \\
& +2 c_{15} n_{1} n_{3}+2 c_{56} n_{2} n_{3}, \\
\Gamma_{12}= & c_{16} n_{1}^{2}+c_{26} n_{2}^{2}+c_{45} n_{3}^{2}+\left(c_{12}+c_{66}\right) n_{1} n_{2} \\
& +\left(c_{14}+c_{56}\right) n_{1} n_{3}+\left(c_{46}+c_{25}\right) n_{2} n_{3}, \\
\Gamma_{13}= & c_{15} n_{1}^{2}+c_{46} n_{2}^{2}+c_{35} n_{3}^{2}+\left(c_{14}+c_{56}\right) n_{1} n_{2} \\
& +\left(c_{13}+c_{55}\right) n_{1} n_{3}+\left(c_{36}+c_{45}\right) n_{2} n_{3}, \\
\Gamma_{22}= & c_{66} n_{1}^{2}+c_{22} n_{2}^{2}+c_{44} n_{3}^{2}+2 c_{26} n_{1} n_{2} \\
& +2 c_{46} n_{1} n_{3}+2 c_{24} n_{2} n_{3}, \\
\Gamma_{23}= & c_{56} n_{1}^{2}+c_{24} n_{2}^{2}+c_{34} n_{3}^{2}+\left(c_{46}+c_{25}\right) n_{1} n_{2} \\
& +\left(c_{36}+c_{45}\right) n_{1} n_{3}+\left(c_{23}+c_{44}\right) n_{2} n_{3}, \\
\Gamma_{33}= & c_{55} n_{1}^{2}+c_{44} n_{2}^{2}+c_{33} n_{3}^{2}+2 c_{45} n_{1} n_{2} \\
& +2 c_{35} n_{1} n_{3}+2 c_{34} n_{2} n_{3},
\end{aligned}
$$

where

$\Gamma_{21}=\Gamma_{12}, ; \quad \Gamma_{31}=\Gamma_{13}, ; \quad \Gamma_{32}=\Gamma_{23}$.

Similarly, the components of the piezoelectric tensor $\gamma_{l}$ may be written as:

$$
\begin{aligned}
\gamma_{l}= & e_{11 l} n_{1}^{2}+e_{22 l} n_{2}^{2}+e_{33 l} n_{3}^{2}+\left(e_{12 l}+e_{21 l}\right) n_{1} n_{2} \\
& +\left(e_{13 l}+e_{31 l}\right) n_{1} n_{3}+\left(e_{23 l}+e_{32 l}\right) n_{2} n_{3}
\end{aligned}
$$

In detail, Eq. (23) may be written in the form:

$$
\begin{aligned}
\gamma_{1}= & e_{11} n_{1}^{2}+e_{26} n_{2}^{2}+e_{35} n_{3}^{2}+\left(e_{16}+e_{21}\right) n_{1} n_{2} \\
& +\left(e_{15}+e_{31}\right) n_{1} n_{3}+\left(e_{25}+e_{36}\right) n_{2} n_{3}, \\
\gamma_{2}= & e_{16} n_{1}^{2}+e_{22} n_{2}^{2}+e_{34} n_{3}^{2}+\left(e_{12}+e_{26}\right) n_{1} n_{2} \\
& +\left(e_{14}+e_{36}\right) n_{1} n_{3}+\left(e_{24}+e_{32}\right) n_{2} n_{3}, \\
\gamma_{3}= & e_{15} n_{1}^{2}+e_{24} n_{2}^{2}+e_{33} n_{3}^{2}+\left(e_{14}+e_{25}\right) n_{1} n_{2} \\
& +\left(e_{13}+e_{35}\right) n_{1} n_{3}+\left(e_{23}+e_{34}\right) n_{2} n_{3} .
\end{aligned}
$$

Similarly, based on Eq. of $(16)_{3}$, one can find:

$$
\begin{aligned}
\varepsilon^{s}= & \varepsilon_{11} n_{1}^{2}+\varepsilon_{22} n_{2}^{2}+\varepsilon_{33} n_{3}^{2}+\left(\varepsilon_{13}+\varepsilon_{31}\right) n_{1} n_{2} \\
& +\left(\varepsilon_{21}+\varepsilon_{12}\right) n_{1} n_{3}+\left(\varepsilon_{23}+\varepsilon_{32}\right) n_{2} n_{3} .
\end{aligned}
$$

The modification of the propagation velocity due to the effect of piezoelectricity can be expressed by changing the stiffness coefficients: the Christoffel tensor can be written in this case as:

$\bar{\Gamma}_{i l}=\bar{c}_{i j k l} n_{j} n_{k}$,

where

$\bar{c}_{i j k l}=c_{i j k l}+\frac{\left(e_{p i j} n_{p}\right)\left(e_{q k l} n_{q}\right)}{\varepsilon_{i j k l} n_{j} n_{k}}$

The terms $\bar{c}_{i j k l}$, known as "stiffened" constants, and they depend on the propagation direction.

To study the propagation of waves along the directions connected with the elements of symmetry of a piezoelectric crystal, we must represent the expressions for the components of tensor $\widetilde{\Gamma}_{k s}$ in case of the system (19) as:

$$
\begin{array}{ll}
\widetilde{\Gamma}_{11}=\Gamma_{11}+\frac{\gamma_{1}^{2}}{\varepsilon^{s}}, & \widetilde{\Gamma}_{12}=\Gamma_{12}+\frac{\gamma_{1} \gamma_{2}}{\varepsilon^{s}}, \\
\widetilde{\Gamma}_{22}=\Gamma_{22}+\frac{\gamma_{2}^{2}}{\varepsilon^{s}}, & \widetilde{\Gamma}_{13}=\Gamma_{13}+\frac{\gamma_{1} \gamma_{3}}{\varepsilon^{s}}, \\
\widetilde{\Gamma}_{33}=\Gamma_{33}+\frac{\gamma_{3}^{2}}{\varepsilon^{s}}, \quad \widetilde{\Gamma}_{23}=\Gamma_{23}+\frac{\gamma_{2} \gamma_{3}}{\varepsilon^{s}} .
\end{array}
$$

\section{Relationship between stress and strain (Elastic constants)}

The coefficients $c_{i j k l}$, which describe the most general linear relationship between the two second rank tensors $T_{i j}$ and $S_{k l}$ are the components of a fourth rank tensors called the elastic stiffness tensor. This proportionality between stress and strain was first enunciated in the stretched string.

A fourth rank tensor has 81 components. However, because $T_{i j}$ and $S_{k l}$ are symmetric tensors, the elastic constants $c_{i j k l}$ remain unchanged under a permutation of $i$ and $j$ or $k$ and $l$ :

$c_{i j k l}=c_{j i l k} ; \quad c_{i j k l}=c_{i j l k}$.

Taking the above symmetries into account, we are left with 36 independent elastic constants, instead of 81 . An unordered pair of indices $(i, j)$ takes on only six distinct values, which we number from 1 to 6 in the following way:

$$
\begin{aligned}
(11) & \leftrightarrow 1, \quad(22) \leftrightarrow 2, \quad(33) \leftrightarrow 3 \\
(23) & \leftrightarrow(32) \leftrightarrow 4, \quad(13) \leftrightarrow(31) \leftrightarrow 5 \\
(12) & \leftrightarrow(21) \leftrightarrow 6 .
\end{aligned}
$$

The independent elastic moduli are thus labeled by only two indices $\alpha$ and $\beta$, ranging from 1 to 6 , and can be arranged in a $(6 \times 6)$ table:

$c_{\alpha \beta}=c_{i j k l} \quad$ with $\quad(\alpha) \leftrightarrow(j k),(\beta) \leftrightarrow(k l)$.

So the $(6 \times 6)$ table of the coefficients $c_{\alpha \beta}$ is symmetric with respect to the main diagonal.

$$
\left(c_{\alpha \beta}\right)=\left|\begin{array}{llllll}
c_{11} & c_{12} & c_{13} & c_{14} & c_{15} & c_{16} \\
c_{21} & c_{22} & c_{23} & c_{24} & c_{25} & c_{26} \\
c_{31} & c_{32} & c_{33} & c_{34} & c_{35} & c_{36} \\
c_{41} & c_{42} & c_{43} & c_{44} & c_{45} & c_{46} \\
c_{51} & c_{52} & c_{53} & c_{54} & c_{55} & c_{56} \\
c_{61} & c_{62} & c_{63} & c_{64} & c_{65} & c_{66}
\end{array}\right| .
$$

The above property reduces the number of independent components to 21 constants, which represents crystals of the triclinic system. 


\section{Tensor formulation of piezoelectricity}

If the strain tensor is symmetric, i.e., $S_{j k}=S_{k j}$, then the tensor $e_{i j k}$ is symmetric with respect to its last two indices:

$e_{i j k}=e_{i k j}$

The number of independent piezoelectric constants is thus reduced from 27 (ordinary third rank tensor) to 18; the pair $(j, k)$ has only 6 distinct values, denoted by the index $\alpha$ according to the convention of equation (1)

$e_{i \alpha}=e_{i j k}, ; \quad(i=1,2,3), ; \quad \alpha=(i, j)=1,2,3 \cdots 6$.

The piezoelectric moduli are arranged in a $3 \times 6$ table (three columns, six rows):

$\left(e_{i \alpha}\right)=\left|\begin{array}{llllll}e_{11} & e_{12} & e_{13} & e_{14} & e_{15} & e_{16} \\ e_{21} & e_{22} & e_{23} & e_{24} & e_{25} & e_{26} \\ e_{31} & e_{32} & e_{33} & e_{34} & e_{35} & e_{36}\end{array}\right|$

\section{Electromechanical Coupling Factor}

There are several ways to define the quantity of the piezoelectric coupling effect. In this section, the electromechanical coupling constants used in this research for bulk acoustic waves and for guided waves are introduced. The phase velocity of a pure elastic unbounded medium is:

$V_{\alpha}=\sqrt{\frac{C}{\rho}}$

and the stiffened phase velocity of a piezoelectric unbounded medium is

$V_{\alpha}^{\prime}=\sqrt{\frac{\bar{C}}{\rho}}=\sqrt{\frac{\bar{C}+\left(e^{2} / \varepsilon^{s}\right)}{\rho}}$,

where $\bar{C}$ is the piezoelectrically stiffened elastic constant and is, in general, calculated from (27). Rewriting (33) yields

$V_{\alpha}^{\prime}=V_{\alpha} \sqrt{1+K^{2}}$

where

$K^{2}=\frac{e^{2}}{C \varepsilon^{s}}=\frac{V_{\alpha}^{\prime 2}+V_{\alpha}^{2}}{V_{\alpha}^{\prime 2}}$

is the coefficient of piezoelectric coupling, which shows the change in acoustic wave propagation due to the effect of piezoelectricity in an unbounded media, and $0 \leqslant K \leqslant 1$.
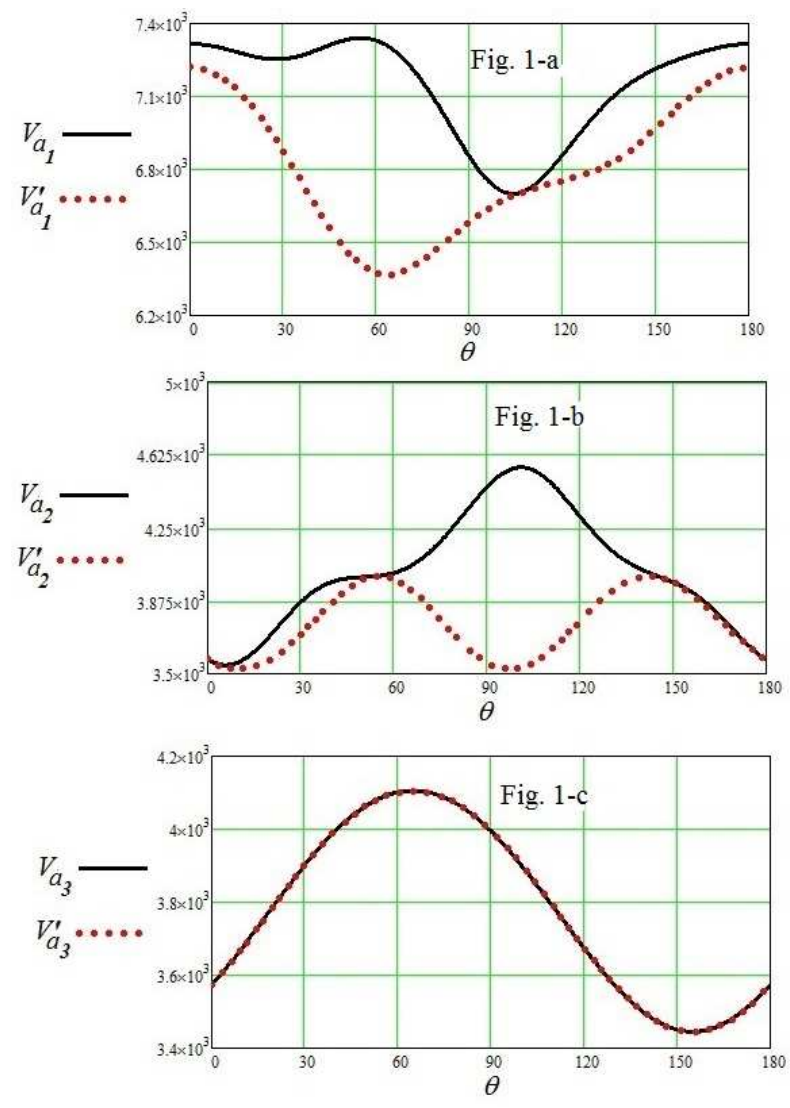

Fig. 1: (1-a), (1-b) and (1-c) represent the velocities of the BAW, denoted by $\left(V_{a 1}, V_{a 2}, V_{a 3}\right)$, as function of $\theta$ for $\mathrm{LiNbO}_{3}$ compared with their counterparts when the piezoelectric constants are zero, denoted by $\left(V_{a 1}^{\prime}, V_{a 2}^{\prime}, V_{a 3}^{\prime}\right)$.

\section{Applications to trigonal symmetry piezoelectric materials (Crystals)}

A Trigonal symmetry crystal has three-fold symmetry around the $x_{3}$-axis. This class includes Lithium Niobate $\left(\mathrm{LiNbO}_{3}\right)$ and Lithium Tantalite $\left(\mathrm{LiTaO}_{3}\right)$. These types of crystals are considered as the most widely used piezoelectric materials, especially for high frequency devices. By using the physical constants of $\left(\mathrm{LiNbO}_{3}\right)$ and $\left(\mathrm{LiTaO}_{3}\right)$ from Table 1 in [9], one may calculate the velocities of BAW propagation $(q P, q S V$ and $q S H)$, which are denoted by $\left(V_{a 1}, V_{a 2}, V_{a 3}\right),\left(V_{b 1}, V_{b 2}, V_{b 3}\right)$ and $\left(V_{c 1}, V_{c 2}, V_{c 3}\right)$, respectively. Additionally, in the special case of neglecting the piezoelectric effect of these materials, the velocities are described as $\left(V_{a 1}^{\prime}, V_{a 2}^{\prime}, V_{a 3}^{\prime}\right),\left(V_{b 1}^{\prime}, V_{b 2}^{\prime}, V_{b 3}^{\prime}\right)$ and $\left(V_{c 1}^{\prime}, V_{c 2}^{\prime}, V_{c 3}^{\prime}\right)$. These velocities are shown graphically in Figures (1) and (2). The electromechanical coupling factors are presented in Figures (3) and (4). 

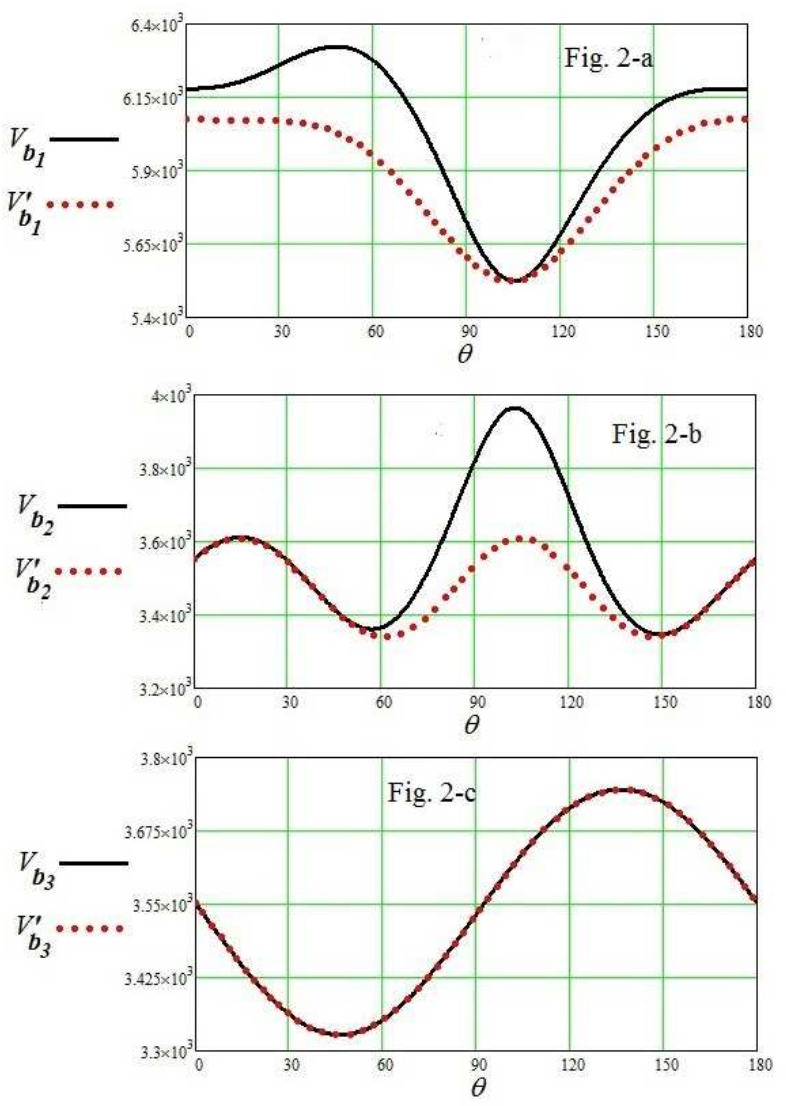

Fig. 2: (2-a), (2-b) and (2-c) represent the velocities of the BAW, denoted by $\left(V_{b 1}, V_{b 2}, V_{b 3}\right)$, as function of $\theta$ for $\mathrm{LiTaO}_{3}$ compared with their counterparts when the piezoelectric constants are zero, denoted by $\left(V_{b 1}^{\prime}, V_{b 2}^{\prime}, V_{b 3}^{\prime}\right)$.

\section{Discussion and the numerical results}

Because of the anisotropy of the trigonal symmetry of crystals, the arbitrary choice of the wave propagation direction, which is required for solving the characteristic equation, is used in Christoffel's equation shown in (18). The corresponding eigenvalues and eigenvectors are the phase velocities of three acoustic modes that are propagating in each direction, one with longitudinal (or quasi-longitudinal) polarization as the $q P$ wave and two with transverse (or quasi-shear vertical and quasi-shear horizontal) polarization as the $q S V$ and $q S H$ waves. Because the corresponding Christoffel's matrix is symmetric, these three eigenvalues are positive real numbers and the acoustic polarizations are mutually orthogonal. The use of a computer-based technique makes it significantly easier to determine the eigenvalues and some of the other acoustic properties. Usually, the first root is the largest one, corresponding to the longitudinal mode, and the other two roots correspond to the fast and slow shear modes. Table (1) lists the physical constants of the anisotropic piezoelectric materials: $\left(\mathrm{LiNbO}_{3}\right),\left(\mathrm{LiTaO}_{3}\right)$ for which the velocities of bulk wave propagation (BAW) are calculated. From Figures (1)-(4), one may conclude the following remarks. In Figures (1-a), (1-b) and (1-c), we show the calculated BAW velocities in $y-z$ plane of piezoelectric $\left(\mathrm{LiNbO}_{3}\right)$ crystal as function $\theta$ (solid curves), which are denoted by $\left(V_{a 1}, V_{a 2}, V_{a 3}\right)$, compared with their counterparts when the piezoelectric and dielectric constants are zero, which are denoted by $\left(V_{a 1}^{\prime}, V_{a 2}^{\prime}, V_{a 3}^{\prime}\right)$ (dot curves). From Fig. (1-a), it is clear that the greatest influence of the piezoelectric effects on the $q P$-wave occurs at $\theta=45^{\circ}$, where $V_{a 1}=3.34 \times 10^{3} \mathrm{Km} / \mathrm{sec}$, and $V_{a 1}^{\prime}=6.241 \times 10^{3} \mathrm{Km} / \mathrm{sec}$.

Furthermore, there is no influence of the piezoelectric effect at $\theta=105^{\circ}$, where $V_{a 1}=V_{a 1}^{\prime} \approx 6.7 \times 10^{3} \mathrm{Km} / \mathrm{sec}$. From Fig. (1-b), for the $q S V$ wave, the greatest influence of the piezoelectric effects occurs at $\theta=100^{\circ}$, where $V_{a 2}=4.56 \times 10^{3} \mathrm{Km} / \mathrm{sec}$, and $V_{a 2}^{\prime}=3.528 \times 10^{3} \mathrm{Km} / \mathrm{sec}$. In addition, there is no influence of the piezoelectric effect at $\theta=55^{\circ}$, where $V_{a 2}=V_{a 2}^{\prime} \approx 4.001 \times 10^{3} \mathrm{Km} / \mathrm{sec}$, as well as over the range of $\theta=145^{\circ}: 180^{\circ}$.

In Figures (2-a), (2-b) and (2-c), the BAW velocities are calculated in the $y-z$ plane of piezoelectric $\left(\mathrm{LiTaO}_{3}\right)$ crystal as function $\theta$ (solid curves), which are denoted by $\left(V_{b 1}, V_{b 2}, V_{b 3}\right)$,and compared with their counterparts when the piezoelectric and dielectric constants are zero, which are denoted by $\left(V_{b 1}^{\prime}, V_{b 2}^{\prime}, V_{b 3}^{\prime}\right)$ (dot curves).

From Fig. (2-a), it is obvious that the greatest influence for the piezoelectric effects in the velocity of the $q P$ wave occurs at $\theta=51^{\circ}$, where $V_{b 1}=6.32 \times 10^{3} \mathrm{Km} / \mathrm{sec}$, and $V_{b 1}^{\prime}=6.016 \times 10^{3} \mathrm{Km} / \mathrm{sec}$, while there is no influence of the piezoelectric effect at $\theta=107^{\circ}$, where $V_{b 1}=V_{b 1}^{\prime} \approx$ $5.527 \times 10^{3} \mathrm{Km} / \mathrm{sec}$.

From Fig. (2-b), for the $q S V$-wave, larger differences between $V_{b 2}$ and $V_{b 2}^{\prime}$ occur for propagation directions $\theta$ ranging from $48^{\circ}: 150^{\circ}$, where $\left(V_{b 2}\right)_{\max }=3.964 \times 10^{3}$ $\mathrm{Km} / \mathrm{sec}$, and $\left(V_{b 2}^{\prime}\right)_{\max }=3.609 \times 10^{3} \mathrm{Km} / \mathrm{sec}$ for $\theta \approx 104^{\circ}$.

For the $q S H$ wave in both of the materials, there is no influence of the piezoelectric and dielectric constants, so the curves are coincide, as shown in Figures (1-c) and (2c).

In Figure (3), the maximum electromechanical coupling factor for the quasi-longitudinal mode (0.494) is shown to be along $\left(60^{\circ}\right)$, and for the quasi-shear vertical mode $(0.633)$ the maximum is along the approximately $100^{\circ}$ direction. The most attractive point is that only one mode is excited, with relatively high coupling values compared with the maximum, at $\left(55^{\circ}\right)$ and $\left(148^{\circ}\right)$ for the quasi-longitudinal mode and at $\left(105^{\circ}\right)$ for the quasi-shear mode. Figure (4) shows that the electromechanical coupling factor for the material $\left(\mathrm{LiTaO}_{3}\right)$ has the maximum values for the quasi-longitudinal mode along $\left(60^{\circ}\right)$ and the maximum for the quasi-shear vertical mode is approximately along $\left(105^{\circ}\right)$. Additionally, one mode is excited at $\left(45^{\circ}\right)$ and $\left(160^{\circ}\right)$ for the quasi-longitudinal 


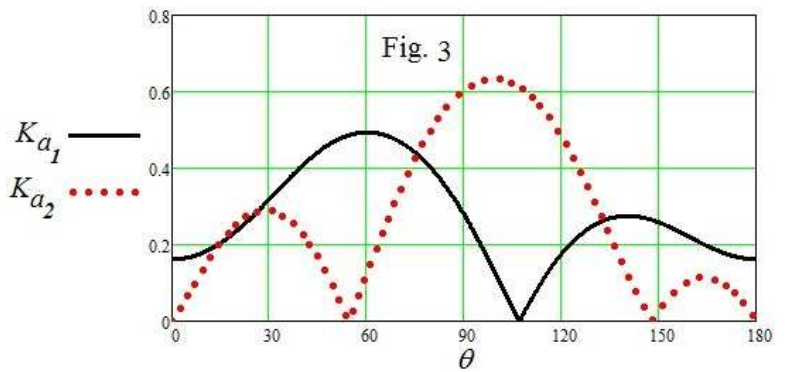

Fig. 3: Represents the electromechanical coupling factors of the BAW, denoted by $\left(K_{a 1}, K_{a 2}\right)$, for the Quasi-longitudinal wave and quasi-vertical waves, respectively, for $\mathrm{LiNbO}_{3}$ as function of $\theta$.

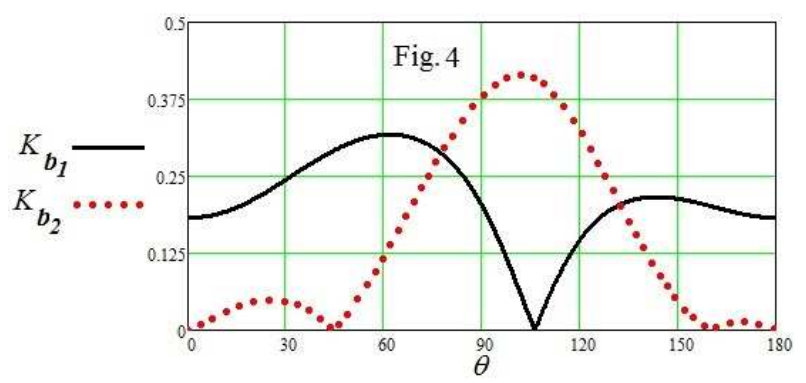

Fig. 4: Represents the electromechanical coupling factors of the BAW, denoted by $\left(K_{a 1}, K_{a 2}\right)$, for the Quasi-longitudinal wave and quasi-vertical waves, respectively, for $\mathrm{LiTaO}_{3}$ as function of $\theta$.

mode and at $\left(105^{\circ}\right)$ for the quasi-shear vertical mode.

Finally, the results show that the electromechanical coupling coefficient of the $q P$ wave is less than that of the $q S V$ wave in both of the two trigonal materials $\left(\mathrm{LiNbO}_{3}\right)$ and $\left(\mathrm{LiTaO}_{3}\right)$.

\section{Conclusions}

The directional dependence of the velocities of bulk wave propagation (BAW) in anisotropic piezoelectric materials was calculated. The velocities were determined for the materials $\left(\mathrm{LiNbO}_{3}\right),\left(\mathrm{LiTaO}_{3}\right)$. In general, the velocities of $q P$ and $q S V$ waves are sensitive to the piezoelectric and anisotropic constants, but the velocity of the $q S H$ wave is not. For the anisotropic case however, there are three general surfaces, that is, one for a quasi-longitudinal wave and two for quasi-shear waves. This property means that the incident and the reflected waves in an anisotropic media can no longer be thought of as purely longitudinal or shear with the appropriate directionally independent
Table 1: Appendix (1)

\begin{tabular}{|c|r|r|r|}
\hline \hline \multicolumn{4}{|c|}{ Lists the physical constants of } \\
\multicolumn{2}{|c|}{$\mathrm{LiNbO}_{3}$ and $\mathrm{LiTaO}_{3}[9]$} \\
\hline \multicolumn{2}{|c|}{ Material } & Lithium & Lithium \\
constants & Niobate & tantalate \\
\hline \multicolumn{2}{|c|}{} & $\mathrm{LiNbO}_{3}$ & $\mathrm{LiTaO}_{3}$ \\
\multicolumn{2}{|c|}{ Trig-3 m } & Trig-3 m \\
\hline Stiffiness & $c_{11}$ & 20.3 & 23.3 \\
$\left(10^{1} 0 \mathrm{~N} / \mathrm{m}^{2}\right)$ & $c_{12}$ & 5.3 & 4.7 \\
& $c_{13}$ & 7.5 & 8.0 \\
& $c_{33}$ & 24.5 & 27.5 \\
& $c_{44}$ & 6.0 & 9.4 \\
& $c_{14}$ & 0.9 & -1.1 \\
\hline Mass density & $\rho$ & 4.7 & 7.45 \\
$\left(10^{3} \mathrm{~kg} / \mathrm{m}^{3}\right)$ & & & \\
\hline Piezoelectric & $e_{22}$ & 2.5 & 1.6 \\
constant & $e_{31}$ & 0.2 & 0.0 \\
$\left(\mathrm{C} / \mathrm{m}^{2}\right)$ & $e_{33}$ & 1.3 & 1.9 \\
& $e_{15}$ & 3.7 & 2.6 \\
\hline Permittivity & $\varepsilon_{11}$ & 38.9 & 36.3 \\
$\left(10^{-11} \mathrm{C} / \mathrm{Vm}\right)$ & $\varepsilon_{33}$ & 25.7 & 38.2 \\
\hline
\end{tabular}

wave speeds. This effect in anisotropic materials also implies that the direction of energy flow (i.e., group velocity) does not, in general, coincide with the normal to the wave front. A computer program was developed to simplify the process of determining the eigenvalues and some of the other acoustic properties of BAW. Such waves are decomposed into finite plane waves propagating along an arbitrary direction in solid. The properties of these waves are determined by the dependence between the propagation direction and the constitutive properties of the medium. Three types of such waves can be distinguished in connection to the three displacement vectors, which determine the acoustic polarization. The three polarization vectors are mutually orthogonal, but in most cases, they are neither perpendicular nor parallel to the propagation direction.

\section{References}

[1] V. Y. Topolov and C. R. Bowen, "From smart materials to piezo-composites", Electro-mechanical properties in composite Based on ferroelectrics, Engineering Materials and Processes, 1-10 (2009).

[2] M. J. P. Musgrave, On an elastodynamic classification of orthorhombic media, Proc. Roy. Soc. Lond., A 4, 401-429 (1981).

[3] R. G. Payton, Elastic Wave Propagation in Transversely Isotropic Media, Martinus Nijhoff, The Hague, (1983).

[4] P. Chadwick, Wave propagation in transversely isotropic elastic media. I. Homogeneous plane waves II. Surface waves, Proc. Roy. Soc. Lond., A 422, 23-66, 67-101 (1989).

[5] V. I. Alshits, P. Chadwick, Concavities on the zonal slowness section of a transversely isotropic elastic material, Wave Motion, 25, 347-359 (1997). 
[6] V. Z. Parton and B. A. Kudryavtsev, "Electromagnetoelasticity: piezo-electrics and electrically conductive solids", J. Breach, NY. (1988).

[7] B. A. Auld, "Acoustic fields and waves", J. Wiley, NY, 1 and 2, (1993).

[8] D. Royer and E. Dieulesaint, "Elastic waves in solids I: Free and guided propagation", Springer-Verlag, Berlin, Germany, (2000).

[9] J. Yang, Special topics in the theory of piezoelectricity, Springer, (2009).

[10] Z. G. Ye, "Handbook of dielectric, piezoelectric and ferroelectric materials Synthesis, properties and applications", Woodhead Publishing Limited, CRC Press, New York, (2008).

[11] R. E. Newnham, "Properties of materials (Anisotropy, Symmetry, Structure)", Oxford University Press, U.K., (2005).

[12] A. G. Khatkevich, The acoustic axes in crystals, . Kristallografiya, 7, 742-747 (1962), (Sov. Phys. Crystallogr. 7, 601-604 (1963)).

[13] V. I. Alshits and J. Lothe, Some basic properties of bulk elastic waves in anisotropic media. Wave Motion, 40, 297313 (2004).

[14] P. Holm and J. Lothe, The topological nature of the polarization field for body waves in anisotropic elastic media. Proc. R. Soc. Lond., A 370, 331-350 (1980).

[15] A. N. Norris, Acoustic axes in elasticity. Wave Motion, 40, 315-328 (2004)

[16] Q. B. Zhou, S. Y. Zhang and Y. K. Lu, "Acoustic anisotropy of piezoelectric $\mathrm{PbB}_{4} \mathrm{O}_{7}$ crystals studied by laser ultrasonics", Materials Science and Engineering, B 83, 249253 (2001).

[17] V. I. Alshits and J. Lothe, "Some basic properties of bulk elastic waves in anisotropic media", Wave Motion, 40, 297313 (2004).

[18] T. C. T. Ting, "On anisotropic elastic materials for which one sheet of the slowness surface is a sphere or a cross-section of a slowness sheet is a circle", Wave Motion, 43, 287-300 (2006).

[19] T. C. T. Ting, "Longitudinal and transverse waves in anisotropic elastic materials", Acta Mechanica, 185, 147-164 (2006).

[20] M. D. Sharma, "Propagation of elastic energy in a general anisotropic medium", Journal of Sound and Vibration, 302, 629-642 (2007).

[21] M. D. Sharma, "Piezoelectric effect on the velocities of waves in an anisotropic piezo-poroelastic medium", Proceedings of the Royal Society A: Mathematical, Physical and Engineering Sciences, 466, 1977-1992 (2010).

[22] E. Langer and S. Selberherr, "Numerical analysis of acoustic wave generation in anisotropic piezoelectric materials", sensors and actuators, 4, 71-76 (1983).

[23] M. Mah and D. R. Schmitt, "Experimental determination of the elastic coefficients of an orthorhombic material", Geophysics, 66, 1217-1225 (2001).

[24] N. F. Cristini, D. Komatitsch, J. M. Carcione and F. Cavallini, "Elastic surface waves in crystals. Part 1: Review of the physics", Ultrasonics, 51, 653-660 (2011).

[25] K. Mauritsson, P. D. Folkow and A. Bostrom, "Dynamic equations for a fully anisotropic elastic plate", Journal of Sound and Vibration, 330, 2640-2654 (2011).
[26] N. I. Ostrosablin, "Purely transverse waves in elastic anisotropic media", J. Appl. Tech. Phys., 46, 129-140 (2005).

[27] A. N. Abd-Alla, A.Y. Al-Hossain, H. Elhaes and M. Ibrahim, Reflection and Refraction of Waves in Nano-Smart Materials: Anisotropic Thermo-Piezoelectric Materials, Journal of Computational and Theoretical Nanoscience, 11, 715-726 (2014).

[28] A. N. Abd-alla, F. A. Alsheikh and A. Y. Al-Hossain, The reflection phenomena of quasi-vertical transverse waves in piezoelectric medium under initial stresses, Meccanica, 47, 731-744 (2012)

[29] A. N. Abd-alla, H. A. Eshaq, and H. Elhaes, The phenomena of reflection and transmission waves in smart nano materials, Journal of Computational and Theoretical Nanoscience, 8, 19 (2011).

[30] A. N. Abd-alla, and F. A. Alsheikh, The effect of the initial stresses on the reflection and transmission of plane quasivertical transverse waves in piezoelectric materials, World Academy Of Science, Engineering and Technology, 50, 660668 (2009).

[31] A. N. Abd-alla, and F.A. Alsheikh, Reflection and refraction of plane quasi-longitudinal waves at an interface of two piezoelectric media under initial stresses", Arch. Appl Mech. 79, 843-857 (2009).

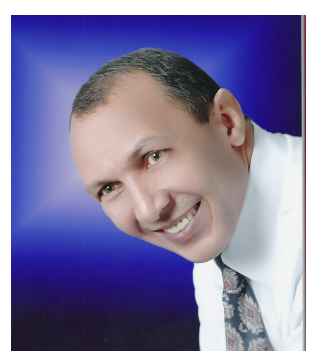

Abo-el-nour Abd-alla received the B.A. degree in Mathematics in 1978, the M.S. degree in 1982 from Assuit University in Egypt. He completed his doctorate in continuum mechanics at the University of Paris (VI), France in 1988 under the super vision of Professor G.A. Maugin. His current research interests include thermal and electromagnetic interactions in deformable solids and linear and nonlinear waves in continuum mechanics. He is a research reviewer in several international journals. He has published over 60 papers in international refereed journals and four books (in Arabic Language).

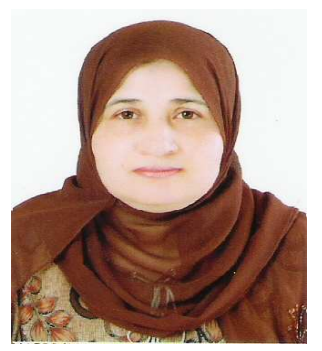

Nadia A. Askar received the B.A. degree in Mathematics in 1992, the M.S. degree in 1996 , Faculty of Girls, Ain Shams University in Egypt. She works as lecturer in Jazan University College of Education Departments of the Mathematics from 2008 until now. She registered for her Ph.D degree in 2010. 